\title{
Zero-a-Seis
}

\section{RECIPROCAL LEARNING: IL CONFRONTO INTERCULTURALE COME DISPOSITIVO PER LA FORMAZIONE DEGLI EDUCATORI - INSIGHTS DA UNA RICERCA TRA ITALIA E STATI UNITI ${ }^{1}$}

\section{Reciprocal learning: intercultural exchange as a tool for continuing professional development of ECEC's educators - Insights from a research in Italy and in the USA}

Reciprocal learning: o confronto intercultural como dispositivo para a formação de educadores Insights de uma pesquisa entre Itália e Estados Unidos

\author{
Piera Maria BRAGA \\ Dipartimento di Scienze Umane per la \\ Formazione "RICCARDO MASSA" \\ Università degli Studi di Milano-Bicocca \\ Milano, Italia \\ piera.braga@unimib.it \\ https://orcid.org/0000-0002-9295-6362
}

Mary Jane MORAN

Department Child and Families studies dell University of Knoxville

Tennessee, USA

mmoran2@utk.edu

https://orcid.org/0000-0003-1882-4326
Chiara Maria BOVE

Dipartimento di Scienze Umane per la Formazione

"RICCARDO MASSA"

Università degli Studi di Milano-Bicocca

Milano, Italia

chiara.bove@unimib.it

https://orcid.org/0000-0003-4818-3777

Robyn BROOKSHIRE

Direttore dell'Early Learning Center for Research and

Practice

University of Knoxville

Tennesee, USA

rbrooks8@utk.edu

https://orcid.org/0000-0001-9525-2865 (D)

A lista completa com informações dos autores está no final do artigo

\section{RIASSUNTO}

Il tema della formazione a una "postura interculturale" fin dai servizi per la prima infanzia è al centro della riflessione contemporanea su come sostenere gli educatori impegnati in contesti educativi multiculturali. Cambiare prospettiva, decentrarsi, provare a vedere i fenomeni educativi da un altro punto di vista, vedere altrimenti sono competenze oggi necessarie per garantire la qualità del lavoro educativo in contesti educativi complessi e multiculturali. Come formare gli educatori che già lavorano nei servizi per l'infanzia a una cultura pedagogica aperta al dialogo interculturale e capace di cogliere nello spiazzamento culturale un'occasione di formazione e di allenamento al pensiero critico-riflessivo?A partire dai dati emersi da una ricerca sulla formazione degli educatori che ha coinvolto alcune educatriciin Italia e negli Stati Uniti, l'articolo propone una riflessione su come i processi di dialogo, confronto e scambio interculturale, mediati da video, possano essere dispositivi interessanti per promuovere lo sviluppo di una postura e non solo di strumenti per l'azione educativa in contesti multiculturali.

PAROLE CHIAVE: Intercultura. Dialogo. Videoricerca. Formazione.

${ }^{1}$ Il contributo è stato concepito congiuntamente dalle autrici, tuttavia: i par. 2, 2.1.1., 2.1.2. sono a cura di Piera Braga; i par. 1,2.1, 2.1.3. sono a cura di Chiara Bove; i par. 2.1.4 e 3 sono stati elaborati da tutte le autrici. Gli estratti di interviste/focus group sono stati individuati nel corpus di dati Italiani e Americani. Ringraziamo tutte le educatrici/insegnanti coinvolte in questo lavoro di ricerca sia in Italia sia negli Stati Uniti. 


\section{ABSTRACT}

The need to foster an "intercultural stance" beginning at the ECE level is a core theme in the current debate on how to support education practitioners working in multicultural settings. Changing one's outlook, decentring, attempting to see educational phenomena from different angles, developing alternative perspectives are required skills for quality educational work in complex and multicultural educational contexts. How can we help ECEC educators to develop an educational culture that is open to intercultural dialogue and views cultural displacement as an opportunity for learning and exercising critical-reflexive thinking? Based on data from a professional development research project with groups of educators in Italy and the United States, this article discusses how video-mediated processes of intercultural dialogue, debate and exchange can help to foster the development of an appropriate educational stance (and not just educational tools) for multicultural settings.

KEYWORDS: Intercultural. Dialogue. Video research. Professional development.

\section{RESUMO}

O tema da formação para uma "postura intercultural" desde os serviços para a primeira infância ${ }^{2}$ está no centro da reflexão contemporânea em como apoiar os/as educadores/as envolvidos/as em contextos educativos multiculturais. Mudar a perspectiva, descentralizar-se, tentar entender os fenômenos educativos de um outro ponto de vista, ver de outra forma, são competências hoje necessárias para garantir a qualidade do trabalho educativo em contextos educativos complexos e multiculturais. Como formar os/as educadores/as que já trabalham nos serviços para a pequena infância para uma cultura pedagógica aberta ao diálogo intercultural e capaz de aproveitar no reposicionamento cultural uma ocasião de formação e exercícios para o pensamento crítico-reflexivo? A partir dos dados obtidos numa pesquisa acerca da formação de educadores/as que envolveu algumas educadoras da Itália e dos Estados Unidos, o artigo propõe uma reflexão a respeito de como os processos de diálogo, de confronto e troca intercultural, mediados por vídeo, podem ser dispositivos interessantes para promover o desenvolvimento de uma postura e não somente como instrumentos de ação educativa em contextos multiculturais.

PALAVRAS-CHAVE: Intercultura. Diálogo. Vídeo-pesquisa. Formação. 


\section{INTRODUZIONE}

Oggi sappiamo che c'è accordo unanime tra ricercatori e decisori politici a livello internazionale sul ruolo dei servizi educativi per l'infanzia (ECEC's settings) nella lotta alla diseguaglianza, alla marginalità e alla povertà educativa (OECD, 2011, 2013; Del Boca, 2010). Contesti paradigmatici per la messa a punto di progetti di accoglienza ed educazione interculturale fin dai primi anni di vita, asili nido e scuole dell'infanzia sono luoghi pubblici in cui i diversi modi di educare, di apprendere e di far star bene i bambini possono incontrarsi, influenzarsi reciprocamente, condividere finalità, obiettivi, strategie. Qui è possibile, fin dai primi anni di vita, formare sguardi consapevoli della complessità dell'intercultura, costruire esperienze di relazione con l'alterità in una dimensione di rispetto dell'altro, generare processi di socializzazione e di costruzione di comunità inclusive, nel rispetto della pluralità di essere e di intendere la famiglia, la cura l'educazione (OECD, 2018). Sappiamo da tempo quanto la sfida dell'interculturalità sia un progetto complesso, ma anche un'occasione per formare una forma mentis aperta, flessibile, plurale. Dobbiamo allo psicologo Luigi Anolli I'aver introdotto nel dibattito italiano sull'educazione la riflessione sulla necessità di accompagnare fin dai primi anni di vita la formazione di "menti multiculturali", capaci di "passare da una cultura all'altra all'interno della propria mente" (ANOLLI, 2011 p.41), allenando i bambini e i ragazzi a sguardi multipli, linguaggi diversificati, visioni del mondo e delle simbologie creative e aperte alla pluralità dei registri.

Un insieme di competenze - comunicative, sociali, relazionali, metodologiche, curricolari, ecc. - strettamente connesse a quelle richieste oggi agli educatori sempre più esposti alla necessità di sviluppare la capacità di riflettere e di apprendere dall'esperienza in una prospettiva di lifelong learning e di capacità di vedere i fenomeni da tanti punti di vista. Questa prospettiva richiama alla necessità di oltrepassare il rischio dell'etnocentrismo - inteso come "radicalizzazione del proprio modo di vedere e capire le cose. Una sorta di provincialismo spaziale e temporale che identifica la totalità dell'esperienza con il segmento (oggi sempre più piccolo) della propria esperienza" (Anolli, 2011 p.41). Sempre più specifici sono i richiami alla necessità di formare gli educatori a competenze riflessive, capacità di confronto intersoggettivo, logica dell'inquiry (DEWEY, 1933 tr.it. 2019) come premesse per il dialogo interculturale. Questa è una condizione oggi trasversale ai contesti educativi e scolastici della società 
contemporanea, e in quanto tale, un potente dispositivo formativo (intendendo con dispositivo quell'apparato simbolico materiale entro cui prende forma l'agire...).

Non sempre approfondita, tuttavia, è la dimensione del "come" formare gli educatori a cogliere nella diversità non tanto un ostacolo, quanto una "provocazione" antropologicamente parlando - che li aiuti ad allargare il punto di vista sul fenomeno ampliando anche il dibattito pedagogico relativo a come sia meglio o preferibile educare i bambini e le bambine (LeVine, New, 2008). Quali contesti possono fungere da laboratorio-interculturale nei servizi per la prima infanzia per formare educatori ed educatrici capaci di "apprendere" dallo spiazzamento culturale, dal dialogo interculturale e dalla differenza di idee, concezioni e pratiche educative? Quali contesti e quali strategie possono aiutarci come ricercatori e formatori ad accompagnare gli educatori a fare dello spiazzamento culturale una palestra pedagogica di formazione?

Proveremo a rispondere a queste domande offrendo alcune indicazioni metodologiche che abbiamo, a nostra volta, appreso lavorando in una prospettiva interculturale e cross culturale in sinergia con un gruppo di ricercatori Americani all'interno di un percorso di ricerca tra Italia e Stati Uniti mediato dall'uso di immagini video come stimolo e medium comunicativo.

L'ipotesi è che lavorando sulla formazione a uno sguardo e a una postura intercultural e possiamo sostenere gli educatori affinché siano modelli indiretti per i bambini e le loro famiglie in contesti sempre più complessi dal punto di vista simbolico, linguistico, culturale, valoriale come i Nidi e le Scuole dell'Infanzia delle società contemporanee. Il fuoco non sarà dunque sull'accoglienza dei bambini migranti e delle loro famiglie, ma sul dialogo interculturale e sullo spiazzamento culturale come strategie e dispositivi per la formazione, nell'ipotesi che la realizzazione piena del primo punto dipenda dalla qualità della preparazione dei professionisti che operano in questi servizi.

Il "potenziale formativo" del confronto tra pratiche, modelli, comportamenti educativi le cui origini culturali sono spesso lontane e non sempre conosciute da chi accoglie i bambini e le famiglie, o a volte addirittura ignorate o fraintese (nei casi più estremi non rispettate), è la tesi che argomenteremo in questo articolo. $E^{\prime}$ importante conoscere la variabilità delle credenze e dei comportamenti educativi agiti nelle diverse comunità culturali, contrastando i rischi oggi in aumento di ignorare o sottostimare questa variabilità - si pensi al rischio di invisibilità che corrono molte famiglie e molti bambini migranti oggi accolti nei servizi per l'infanzia delle nostre comunità occidentali (BOVE; SHARMAHD, 2020) - ma è ancora più importante accorgersi di come reagiscono individui di altre parti del mondo a questi modelli, imparando a riconoscere le 
provocazioni che questi modelli generano in chi li osserva da vicino o da lontano in termini di occasioni o diritti di essere vistie rispettati nella diversità. Lo spiazzamento culturale è un diritto reciproco - in termini di etica della relazione - ma anche un'occasione di apprendimento per entrambi, ma in primis per chi è impegnato a livello professionale come educatore o educatrice.

Ipotizziamo che la complessità linguistica, valoriale, religiosa, culturale che si osserva oggi nei contesti educativi per l'infanzia - veri "laboratori di incontro e dialogo" - richieda educatrici e educatori preparati e allenati a fare del confronto interculturale e dell'incontro con l'alterità i loro principi pedagogici guida, senza i quali è impossibile costruire comunità educanti effettivamente e autenticamente inclusive, democratiche, interculturali.

Il nostro obiettivo è contribuire al dibattito sull'innovazione dei metodi per la formazione in servizio degli educatori - tema che è stato al centro di esperienze di ricerca recenti che hanno coinvolto il nostro gruppo (BOVE, JENSEN, et.al. 2018) offrendo spunti e metodi per costruire contesti che favoriscano i complessi dinamismi dell'esperienza del dialogo interculturale - anche a distanza per una questione di sostenibilità oggi importante in un momento di ristrettezze economiche - intese come "palestre" di allenamento al decentramento e alla capacità di cogliere la ricchezza dell'incontro con la diversità.

\section{LA RICERCA}

La ricerca a cui facciamo riferimento ${ }^{3}$ ha coinvolto le educatrici di due Nidi Universitari (il nido Bambini Bicocca dell'Università di Milano-Bicocca e il Nido di

\footnotetext{
${ }^{3}$ La ricerca, dal titolo "Teachers' Behavior Analysis in Infant-Toddler Centers: Instruments and Methods for Training within a Cross-National Research Project, ha coinvolto i due Nidi Universitari dell'Università di Milano-Bicocca (Dipartimento di Scienze Umane per la Formazione "Riccardo Massa") e I'Università del Tennessee/Knoxville (College of Education, Health and Human Sciences/Department of Child and Family Studies). Responsabili scientifici del Progetto: Mary Jane Moran e Susanna Mantovani; coordinatori del Progetto in Italia Piera Braga e Chiara Bove. Altri componenti del team di ricerca: Robyn Brookshire e Silvia Cescato. La ricerca è stata presentata nei seguenti articoli e saggi a cui rimandiamo per approfondimenti: BRAGA P. (2009, a cura di), Promuovere consapevolezza. Esperienze di formazione tra ricerca e pratica educativa, Junior, Azzano San Paolo, Bergamo; BOVE, C., BRAGA, P. (2014). "Il Nido come laboratorio di ricerca: osservare e osservar(si) nei contesti educativi per l'infanzia". In F.L. Zaninelli (a cura di), Contesti e temi educativi zero-tre anni (pp. 273-295). Parma: Junior- Spaggiari edizioni srl.; BOVE C., BRAGA P., CESCATO S. (2014), "Semantiche personali e processi riflessivi. Analisi di una storia di formazione", Studium Educationis, 1, Febbraio 2014, 75-84; BOVE, C., BRAGA, P. MANTOVANI, S. "Videoricerca e formazione. Un approccio interculturale". In Bobbio, A., Traverso, A. (a cura di) Contributi per una pedagogia dell'infanzia Teorie, modelli, ricerche. ETS edizioni, Pisa, 2016. Pp.147-173; BRAGA., BOVE C., "Osservare e osservarsi al nido", Bambini, n.8, Ottobre, 2014, pp.20-23; CESCATO S., BOVE C., BRAGA P. (2015), "Video, formazione e consapevolezza. Intrecci metodologici", in «Form@re», XV, 2, 61-74; BOVE C., (2016), "Le parole nella relazione con i bambini: osservare il "linguaggio in azione" al nido", in S.
} 
Knoxville, Università del Tennessee ${ }^{4}$ ) allo scopo di studiare modelli innovativi di formazione in servizio degli educatori in una prospettiva di sostenibilità e scambio interculturale (MORAN et al.2017; Moran, et.al. 2018). La ricerca si è svolta dal 2009 al 2013 e gli obiettivi sono così sintetizzabili: attraverso l'osservazione di una giornata tipo in entrambi i contesti cogliere elementi di somiglianza e differenza a livello di svolgimento, conduzione, stile educativo, curricolo; coinvolgere gli educatori di entrambi i Nidi in un processo sistematico, rigoroso, ricorsivo di analisi e revisione critica del proprio comportamento e di quello altrui, in Italia e negli Stati Uniti e viceversa; stimolare il dialogo interculturale tra gruppi di educatrici di contesti culturali e linguistici diversi - Iontani per posizione geografica, storia, tradizione pedagogica, approccio metodologico - in una prospettiva di apprendimento reciproco (reciprocal learning) (BRAGA, 2009; BRAGA, BOVE, 2014).

A livello meta la ricerca ha avuto come finalità la messa a punto di metodi innovativi per la formazione in servizio degli educatori. La ricerca ha infatti previsto due fasi interconnesse: in una prima si è videoregistrata una giornata di ciascuna educatrice di una sezione in entrambi i nidi e, nella seconda fase, si sono organizzati diversi momenti di riflessione e confronto tra le educatrici,intra e inter-contesto, sia a livello individuale sia in gruppo;i video sono stati utilizzati come stimolo dapprima per rivedersi e auto-valutarsi - attivando le educatrici in un processo di auto-valutazione finalizzato a prendere coscienza dei propri modelli educativi e delle pedagogieimplicite - e, successivamente, per vedere le colleghe dell'altro Nido ripensando ai loro e ai propri comportamenti educativi e modelli (ALTET, 1996).

Parallelamente le educatrici riprese nelle videoregistrazioni - cosiddette "focalteachers" (3 in Italia, 2 negli Stati Uniti) - sono state coinvolte in un percorso di riflessione critica stimolata a posteriori attraverso un'intervista, successivamente riproposta a più riprese, per consentire l'avvio di un percorso di riflessione ricorsiva e approfondita sul proprio agire che tenesse conto delle evoluzioni nel modo di descrivere

Mantovani, Silva C., Freschi E. (a cura di) Didattica e Nido d'infanzia. Metodi e pratiche di intervento educativo, Junior-Spaggiari, Parma; MORAN M.J., BOVE C., BROOKSHIRE R., BRAGA, P., MANTOVANI, S. (2017), "Learning from each other: The design and implementation of a cross-cultural research and professional development model in Italian and U.S. toddler classrooms", Teaching and Teacher Education, 63, pp. 1-11; MORAN, M.J., BROOKSHIRE, R., BOVE C.,BRAGA P., MANTOVANI S. (2018). "Co-Constructed Research Design: Lessons on Equivalency and Teacher Participation in a US-Italian Professional Development Study", in Apkovo S.M., Moran, M.J, Brookshire, R. (eds), Collaborative Cross-Cultural Research Methodologies in Early Care and Education Contexts, Taylor\& Francis,133-149, New York.

${ }^{4}$ Il Nido dell'Università di Milano-Bicocca può accogliere 64 bambini, ed è articolato in 3 sezioni (una sezione che accoglie 14 bambini piccoli, dai 3 ai 12 mesi, e due sezioni di età mista medi (12-24 mesi) e grandi (24-36 mesi). Il Nido dell'Università del Tennessee- Knoxville può accogliere 40 bambini, ed è articolato in tre sezioni di età omogenea. 
il proprio comportamento e di dare senso al proprio agire. La riflessione si è svolta a livello individuale e successivamente in gruppo, con le colleghe del proprio e dell'altro contesto, poi ancora a livello individuale generando un processo ricorsivo di analisi e revisione critica delle immagini, esplicitazione dei propri presupposti pedagogici, eccetera. Tra la revisione individuale delle immagini e quelle di gruppo, si sono create occasioni di scambio e confronto a livello interculturale mediate dalle tecnologie (conversazioni skype, scambio di video-clip, e-mail).

Al di là dell'articolazione metodologica del progetto, esito del lavoro cross culturale e di collaborazione intensa tra le équipe di ricerca dei due paesi - le cui potenzialità sono state presentate in Moran et. al.2018 - ci sembra interessante soffermarci sulla dimensione interculturale dello scambio che si è generata sia a livello di gruppo di educatrici appartenenti allo stesso Nido (colleghe di sezioni diverse o della stessa sezione) sia tra i due Nidi (nido Italiano e nido americano), mediato dall'uso delle videoregistrazioni usate come "testo multimediale" o reattivo (TOBIN, 1989; 2009) per avviare processi di confronto e dialogo interculturale e cross culturale a partire da uno stimolo condiviso: le educatrici che vedono la stessa clip-video, dell'uno o dell'altro contesto, e ne discutono. Diversamente dal metodo di etnografia visuale e multivocale introdotto in ambito educativo da J. Tobin $(1989,2009)$ e che ha molto ispirato il disegno metodologico del progetto, in questa ricerca le clip-video sono state utilizzate più come testi-descrittivi e dunque insieme di comportamenti educativi da analizzare sia alternando visioni micro e macro e intrecciando la descrizione dei comportamenti alla riflessione sulle premesse o sugli impliciti pedagogici, che come edited-video come nella proposta originale di Tobin (TOBIN, 1989, tr. 2000; 2009, tr. 2010; TOBIN, MANTOVANI, BOVE, 2010). Ci sembrava - come abbiamo avuto modo di discutere anche in altri contributi (BOVE, BRAGA, MANTOVANI, 2016) - che l'incontro interculturale tra le educatrici mediato dalle immagini potesse essere un metodo innovativo e protetto per stimolare il confronto autentico tra le protagoniste, in una prospettiva di apprendimento reciproco e di esposizione alle proprie e altrui pratiche quotidiane.

Si ipotizzava che l'esposizione delle educatrici italiane alle pratiche educative agite dalle colleghe americane, e viceversa, avrebbe innescato, sostenuta da un dispositivo predisposto ad hoc, un processo di osservazione, analisi, riflessione critica sulle pratiche proprie e altrui più decentrato e meno condizionato da automatismi interpretativi e di azione legati all'abitudine (BOVE, BRAGA, MANTOVANI, 2016, p. 153). 
La dimensione processuale, ricorsiva, estesa nel tempo, intrecciando I'osservazione mediata dalle videoregistrazioni e la riflessione sui propri e gli altrui comportamenti, costituiscono gli elementi di innovazione metodologica del progetto da cui emergono spunti per ragionare su come accompagnare gli educatori in processi di revisione critica e ampliamento dello sguardo. $E^{\prime}$ interessante rilevare quanto il dispositivo del confronto interculturale abbia innescato processi di revisione critica dei temi portanti del progetto pedagogico del servizio che i gruppi di lavoro nei due contesti stanno riprendendo e approfondendo separatamente.

Vediamo nei prossimi paragrafi alcuni esempi dai dati da cui dedurre indicazioni per la messa a punto di contesti e metodi per innovare la formazione interculturale degli educatori in una prospettiva di formazione on the job e ricerca-formazione.

\section{Considerazioni dai dati}

I dati emersi dal percorso di ricerca sono diversi tra loro e articolati su due livelli: metodologico e di contenuto. Nel contesto Italiano, a cui facciamo riferimento in particolare in questo articolo, la ricerca ha fatto emergere interessanti direzioni di approfondimento e riconsiderazione di temi quali il rapporto con le famiglie e le pratiche di accoglienza (inserimento/ambientamento), il significato delle regole, I'autonomia, I'uso del linguaggio nella quotidianità del nido, il ruolo dell'adulto nelle relazioni tra i bambini. Temi emersi talvolta per riconfermare le premesse pedagogiche che informano l'agire quotidiano, talvolta per problematizzare le pratiche abituali ampliando la prospettiva e le possibili strategie di intervento educativo, talvolta per introdurre prospettive diverse (MORAN, BOVE, et.al. 2017).

Senza pretese di esaustività, ci soffermiamo di seguito su tre esempi dai dati che bene esemplificano alcune acquisizioni metodologiche che riteniemo interessanti per ampliare il dibattito sull'innovazione nella formazione interculturale degli educatori: il primo illustra il potenziale formativo dell'auto-osservazione mediata da video e dalla possibilità di riveder(si) sia individualmente sia con altri (osservare altrimenti); il secondo illustra il potenziale formativo dell'osservazione combinata con altri strumenti di rilevazione delle rappresentazioni e dei significati come medium per ampliare il modo con cui le educatrici "pensano" al curriculum (pensare altrimenti); il terzo illustra un tema emerso dall'analisi dei dati che, inaspettatamente, ha aperto prospettive interessanti per la formazione interculturale degli educatori (parlare altrimenti). Si tratta di esempi che abbiamo in parte discusso in precedenti lavori (BRAGA, 2009; MORAN, 
BOVE, ET.AL. 2017; BOVE, BRAGA, 2014; BOVE, 2016), ma che qui rileggiamo come dispositivi per la formazione di uno sguardo interculturale.

\section{Osservare altrimenti}

Il primo esempio ci consente di ragionare sul potenziale formativo dell'intreccio tra osservazione, auto-osservazione e riflessione sulle pedagogie implicite mediato dall'uso del video "come dato" (Goldman, et.al. 2007) o testo da analizzare per comprendere e far emergere le "ragioni" o pedagogie implicite che orientano l'agire educativo. L'uso del video ha caratterizzato tutto il processo di ricerca, ma si è rilevato particolarmente promettente come medium per la formazione interculturale quando è stato proposto alle educatrici come "stimolo" per la riflessione individuale. La prima fase della ricerca, infatti, ha coinvolto le educatrici protagoniste dei filmati in alcuni momenti di revisione individuale dei propri filmati proposti in modo ricorsivo. Ciascuna educatrice è stata invitata a rivedere individualmente e per due volte l'intero video della loro giornata allo scopo di attivare una rielaborazione individuale dell'esperienza a partire dall'auto-osservazione: nel corso della prima revisione le educatrici sono state invitate a esprimersi liberamente sul proprio video, annotando le proprie considerazioni e segnalando le scene da eliminare (diritto di veto); nella seconda fase, invece, la riflessione è stata guidata da uno strumento messo a punto dai ricercatori come "guida" alla revisione individuale. Entrambe le fasi sono state poi accompagnate da momenti di narrazione-ricostruttiva tramite intervista, al fine di sollecitare la rievocazione di eventi/situazioni e dei pensieri che li hanno orientati. Il video (con e senza il ricorso alla forza narrativa del montaggio)è stato utilizzato sia come strumento di analisi dei contesti e delle pratiche sia come reattivo per analizzare le rappresentazioni, per ritornare su ciò che è accaduto in una determinata situazione secondo le logiche del video-feedback (Tochon, 2007). Le considerazioni (orali e scritte) fatte dalle educatrici protagoniste nel corso della revisione individuale dei filmati e tratte dalla prima intervista descrivono molto bene il valore e le potenzialità euristiche di questi momenti di auto-osservazione e di riflessione individuale sulla propria esperienza e sul proprio operato. Quello che ci sembra interessante sottolineare è che si tratta di un "esito soprendente" per le educatrici Italiane coinvolte e in un certo senso "inatteso e spiazzante" e - pertanto - interessante: nella tradizione pedagogica italiana, infatti, siamo soliti pensare alla formazione degli educatori come esperienza condivisa, di gruppo, collegiale. Rare sono le occasioni in cui gli educatori sono invitati a "sostare" 
sui propri comoprtamenti o convinzioni educative da soli. Il gruppo, infatti, è concepito come "contesto-ideale" per la formazione degli insegnanti e, conseguentemente, tutti gli insegnanti e gli educatori si aspettano che la formazione sia erogata in contesti di gruppi. Senza negare il valore del confronto collegiale e del gruppo, che nella tradizione pedagogica italiana costituise il contesto elettivo della formazione in servizio (Mantovani, 2007), emerge dall'analisi dei dati la potenzialità - per noi - inattesa del lavoro individuale. Chiedere alle educatrici Italiane di "rivedersi individualmente" annotando le proprie considerazioni e, soprattutto, "scrivendo" ha funzionato da stimolo per promuovere processi di spiazzamento culturale e formativo. Per alcune si è trattata di un'esperienza insolita, molto spiazzante; per altre è stata un'esperienza interessante e arricchente, ma non priva di difficoltà. Tutte sottolineano la fatica e I'utilità delle revisioni individuali, enfatizzando quanto sia la revisione libera, sia quella più strutturata guidata da uno strumento di autovalutazione, insieme al coordinatore-ricercatore, siano stati momenti "inattesi" (sorprendenti) del processo di ricerca. In particolare, la fatica sembra attribuita a due questioni: l'impatto del rivedersi e la tendenza a notare prima gli aspetti critici e negativi e poi quelli positivi.

Riguardare il filmato è stato molto utile [...] e l'impatto nel rivedersi è stato abbastanza forte [...] ho notato subito le cose che non andavano, non quelle positive. Quelle positive forse le ho notate di più in seguito, facendo la seconda revisione. (Educatrice, Italia).

La seconda revisione del filmato è più strutturata, guidata da una procedura che sollecita la segmentazione e l'autovalutazione degli eventi ma anche dalla presenza del ricercatore: è una sorta di pensiero "ad alta voce" dove la condivisione della riflessione, la presenza dell'altro (sia esso un collega, il coordinatore, il ricercatore, il gruppo di lavoro) consente di evitare che il momento di auto-osservazione individuale diventi troppo autocentrato, che diventi una sorta di "autocoscienza" che sconfina nell'autoreferenzialità. La procedura di codifica e autovalutazione favorisce la presa di coscienza e l'esplicitazione delle motivazioni di una impressione o di un giudizio (positivo o negativo) su una situazione o su una scelta di azione («non mi piaccio, ero agitata, non sono io...»), aiuta ad argomentare le impressioni e i giudizi su di sé e sulle situazioni oltre che sui bambini, aiuta ad esprimere considerazioni più eterocentrate e meno autoriferite, a riflettere sul proprio lavoro in maniera più distanziata e critica.

Un conto è fare un filmato perché vuoi osservare i bambini, un conto è fare un filmato su te stessa, su come ti muovi perché ... tendi sempre a guardare i bambini...; è difficile però è una difficoltà positiva. Dovevi esplicitare, ti obbligava a esplicitare il perché davo quel punteggio, perché mi piaceva quella scena. Non è semplice esplicitare perché mi piace, perché ci tengo molto che questa scena 
venga condivisa: questa è stata la cosa che forse mi è stata forse più utile (...). Non è solo un dire mi piace, non mi piace, ma anche il perché (Educatrice, Italia).

Emerge una differenza interessante tra quando ci si rivede per la prima volta e per la seconda volta, tra quando ci si rivede da soli o con gli altri. E il tempo molto disteso e la ricorsività della revisione sono stati due aspetti importanti di questo processo.

\begin{abstract}
La prima volta che ho rivisto il filmato ero molto concentrata su di me, dopo ho provato ad avere una visione ... di insieme. Nella seconda revisione mi sono accorta di essere riuscita a guardare un po' di più la situazione generale ... però di questo passaggio ... me ne sono accorta dopo. La prima volta è stata una carrellata, ti focalizzi di più sulle scene da eliminare, la seconda volta invece ci siamo focalizzate proprio scena per scena, minuto per minuto, quindi è stata una cosa molto approfondita. Questa parte è stata la più interessante, è stato difficile anche emotivamente, soprattutto la fase del rivedersi ... molto, molto forte, però sicuramente molto utile. Ti rivedi da fuori, ti accorgi di tantissime cose che da dentro non vedi: quando I'ho visto la prima volta ho detto 'no, questo non va, questo non va' (...) però il fatto di rivedersi, di fermarsi a riflettere, ti aiuta ancora di più a stare attenta... Il fatto di dare un punteggio alla situazione: devi avere diversi occhi, su di te, sui bambini ..., è difficile però è una difficoltà positiva. Dovevi esplicitare (..) perché davo quel punteggio, perché mi piaceva quella scena. Esplicitare queste cose non è semplice ... perché sei dentro, è difficile però è interessante (Educatrice, Italia).
\end{abstract}

E' la potenza del rivedersi (BRAGA, BOVE 2014), è la valenza formativa dell'autoosservazione come strumento di riflessione sul proprio agire e sulle idee che orientano e sostengono l'azione educativa quotidiana. Emerge la potenzialità formativa della revisione individuale, e del tempo disteso dedicato a questo momento dove, rivedendo le immagini, le educatrici protagoniste diventano spettatrici e commentatrici di se stesse; e quindi l'importanza di sostare su questo momento di auto-osservazione e rielaborazione individuale dell'esperienza come premessa e preparazione più consapevole al confronto di gruppo. Una fase importante perché prima di tutto accredita il contributo e il pensiero del singolo, ne sollecita l'attivazione riflessiva e promuove l'acquisizione di un atteggiamento sostanzialmente progettuale e "di ricerca"; apre inoltre uno spazio di riflessione personale, autonomo, che prepara al confronto con le colleghe (che resta sempre molto atteso, perlomeno da noi), ma non delega al gruppo la rielaborazione critica, la ricerca delle domande, dei significati possibili di una situazione.

Le educatrici sono state sollecitate a rintracciare gli impliciti, le ragioni delle loro scelte e valutazioni (mi piace, non mi piace e perché? Vorrei, non vorrei condividerlo con le altre e perché?), i loro riferimenti e modelli, le esperienze che informano il loro agire (per esempio una domanda che veniva posta nel corso focus group, ha fatto emergere delle considerazioni e discussioni interessanti a questo riguardo...). Abbiamo 
chiesto alle educatrici uno sforzo di rievocazione e di esplicitazione, di ricostruzione critica dei propri comportamenti e saperi, per indagare soprattutto l'inatteso, ciò che sorprende e non solo ciò che conferma le aspettative le immagini che le educatrici hanno del proprio agire, sollecita nuove consapevolezze: «C'è qualcosa che avete visto nel filmato che non ti aspettavi di vedere? C'è qualcosa che ti aspettavi di vedere nel filmato, ma che non hai visto? Qualcosa che ti ha sorpreso?».

E sono soprattutto le sequenze che non si aspettavano di vedere, piuttosto che le aspettative confermate, che sembrano essere maggiormente utili per acquisire un maggiore autocontrollo. Riportiamo alcune considerazioni delle educatrici italiane:

Mi aspettavo di meno il lavoro individuale, l'approfondire individualmente è piacevole nel senso che è una ricchezza che non tutti i giorni dedichiamo...

Non mi aspettavo tutta la [...] cioè quello che è servito a me. [...] Non mi aspettavo che questo lavoro fosse utile per me».

Mi ha colpito il modo in cui mi muovevo nello spazio, mi muovevo tanto, e rivedendolo nel filmato I'ho notato ancora di più; anche il fatto di tenere la voce alta [...]. Allora adesso forse riesco a controllarlo un po' in alcuni momenti quando me ne accorgo, riesco a rendermene conto, ad usare un altro tono di voce...anche perché lo vedo riflesso nei bambini [...]. Non mi aspettavo che si vedesse così tanto.

Ho dato un punteggio alto [a quella scena] perché avevo la calma; quello è forse il migliore esempio di buon comportamento, sono tranquilla e questo passa ai bambini $[\ldots]$ ho aspettato, li ho condotti con la voce.

[...] cioè quello che mi sono portata dietro di questo lavoro è che mi ha dato $\mathrm{i}$ punti per ripartire su quali sono le mie "difficoltà" [...] rivederti ti aiuta a capire, a riprogettare. [...] Ho pensato che faccio fatica, ho delle difficoltà a dare attenzione a tutti, a tenere il gruppo. Questo è un po' il punto su cui devo lavorare e... su cui mi piacerebbe anche avere un confronto con le altre.

Poi c'è I'attesa della condivisione, perché sei interessata a sapere come la vede la collega, cioè comunque si lavora insieme 'io l'ho visto così, e tu?

Adesso sono curiosa di come possa avere un proseguo anche dal confronto con gli altri...

La riflessione sull'esperienza si arricchisce ed evolve attraverso il confronto intersoggettivo e il lavoro individuale prepara e favorisce un accesso più attivo e consapevole al confronto di gruppo.

Se questo sostare a lungo nella fase della revisione e auto-valutazione individuale del video è risultata più familiare alle colleghe nordamericane, tradizionalmente più abituate a una metodologia di lavoro centrata sulla dimensione individuale o del confronto con la singola collega di sezione, meno sulla dimensione collegiale della progettazione e della formazione; la fase della discussione in gruppo dei filmati è stata 
particolarmente innovativa e proficua per le educatrici americane che hanno iniziato a interrogarsi sulla necessità di creare più occasioni di confronto e discussione collegiali.

Il tema dell'equilibrio tra dimensione individuale e di gruppo della riflessione sull'esperienza ha sollecitato interessanti riflessioni e acquisizioni, anche per la ricerca sulla formazione. Abbiamo ragionato su come integrare meglio queste due dimensioni, prevedendo anche percorsi di attivazione individuale, considerando la sostenibilità dei percorsi formativi, il turn over che caratterizza la composizione dei gruppi di lavoro/ ricambio intergenerazionale, la necessità di lavorare sulle risorse di tutti, pur sempre sullo sfondo di una cultura della collaborazione o del lavoro collegiale che è tipica della comunità dei servizi dell'infanzia in particolare nella tradizione italiana. Le educatrici italiane hanno sviluppato maggior familiarità con pratiche di osservazione e analisi individuali e in coppia. Come ci si prepara al momento del confronto di gruppo per rendere le riunioni $e$ in particolare i momenti/percorsi formativi più realmente partecipati, per non perdere tempo, per attivare e dare voce a tutti, per raccogliere le idee prima di condividerle con gli altri?

La scrittura, per esempio, è uno strumento prezioso anche come operazione preliminare al confronto di gruppo, che ferma e mette in moto i "pensieri", che dà voce a tutti, che invita e legittima tutti ad esprimersi. Scrivere i pensieri sollecitati da un materiale osservativo (video o scritto) innesca e sostiene la riflessione, come rilevano sostengono le educatrici protagoniste durante un'intervista:

Anche scrivere, è una difficoltà che però ti obbliga a dare un nome a quella cosa che tu vedi, mentre a volte dici «sì mi piace, non mi piace», invece no, devi dare un nome a questa cosa e il fatto di farlo da sola ti obbliga a farlo, sì è stato più utile altrimenti ci saremmo influenzate (Educatrice, Italia).

$\mathrm{E}^{\prime}$ stato molto utile raccogliere i pensieri per iscritto dopo la visione dei video, per non perdersi nel verbale (Educatrice, Italia).

\section{Pensare altrimenti}

Il processo di recupero delle proprie pedagogie implicite, indotto e sostenuto dal confronto interculturale e dall'intreccio tra osservazione e strumenti che strutturano la riflessione è servito talvolta per riconfermare a distanza di tempo la proprie convinzioni, talvolta per problematizzare o per introdurre prospettive "insolite" o "nuovi modi di vedere i fenomeni educativi", ma comunque sempre per chiarire a sè stessi e agli altri (e viceversa) ciò che si pensa e che orienta ciò che si fa e come lo si fa. Nelle diverse fasi della ricerca attraverso il confronto (inter e intraculturale) le educatrici protagoniste della ricerca, insieme alle colleghe e ai ricercatori, sono state sollecitate a ritornare agli 
assunti che orientano il progetto educativo dei servizi e le azioni che quotidianamente lo interpretano, a "riprendere in mano" temi e questioni che stanno emergendo anche da altri percorsi formativi e ricerche perché particolarmente pregnanti e attuali, ma che nella cornice dell'impianto interculturale balzano molto velocemente in primo piano e stanno aprendo interessanti piste di approfondimento che stiamo continuando ad esplorare con modalità diverse.

Soffermiamoci su un esempio emergente dai dati relativo alla gestione del gruppo dei bambini da cui emerge, nuovamente, il tema del rapporto tra individuo e gruppo (come abbiamo visto nell'esempio precedente). Temi quali la conduzione del gruppo dei bambini, le strategie di interazione e mediazione dei conflitti tra bambini, il ruolo e intervento dell'adulto delle diverse attività (più o meno diretto/indiretto), il modo in cui si parla con i bambini, il senso delle regole, l'autonomia, sono al centro degli interrogativi degli educatori che lavorano in contesti interculturali. Non si tratta di temi nuovi per il dibattito pedagogico sui servizi per l'infanzia in Italia, ma è la modalità con la quale emergono che risulta innovativa e funziona da stimolo per mettere in campo riflessioni, intenzionalità e progettualità rinnovate, azioni nuove. Innovare, non significa necessariamente stravolgere. Talvolta significa confermare e chiarire gli assunti e i criteri che determinano le modalità di azione e sentirsi parte di una comunità educativa. Il processo di immersione e differenziazione che si attiva quando gli educatori vedono il filmato delle colleghe americane, e viceversa, stabilendo un contatto con un'altra cultura (anche in senso metaforico: sperimentando che cosa significa essere là) consente processi di ampliamento delle proprie interpretazioni interessanti in prospettiva formativa.

Le parole delle educatrici dei due contesti, che si confrontano a partire dalla visione dei reciproci filmati, descrivono bene/ben esemplificano questi movimenti di pensiero e ripensamento. Alcuni commenti delle educatrici italiane:

Mi sembra che quando un'educatrice nel filmato americano parla, parli sempre a un bambino... lei focalizza sempre l'attenzione su un bambino per volta... noi sempre ampliamo la discussione al gruppo per coinvolgerli tutti... per noi lo spuntino è un momento di convivialità.

A me è piaciuto l'ordine... [...]... appena il bambino sta per alzarsi gli dice <mettilo nel cestino...mi ha colpito la tempestività: appena un oggetto è spostato dice $<$ metti nel cestino >... Però non dice metti a posto dice <se avete finito metteteli nel cestino, grazie $>$.

Nel vedere con le colleghe il filmato americano ho sentito tanto il gruppo di lavoro in cui sono inserita, e mi ha confermato come crediamo in aspetti pedagogici che tutti i giorni si materializzano, un lavoro comune.

Vedere il filmato americano è stato sorprendente, all'inizio I'ho guardato dal mio punto di vista cercando somiglianze e differenze, poi, alla fine del primo incontro, ho provato a spostarmi dal mio punto di vista per capire meglio la loro prospettiva. 
Il concetto di fare le cose in comunità, fare le cose insieme probabilmente è una cosa culturale nostra, cosa che loro non hanno. Noi mangiamo insieme, noi ci aspettiamo per mangiare, noi andiamo a lavarci le mani insieme, andiamo a dormire tutti insieme, siamo vicini [...] il vedere che non si aspettano per mangiare e soprattutto dormono Iontani ci ha un po' destabilizzato. Mi sto interrogando su questo...

Alcuni commenti delle educatrici americane:

$\mathrm{E}^{\prime}$ come se l'educatrice dicesse ai bambini 'io sto qui seduta e vi guardo'... credo che sia diverso da quello che cerchiamo di fare noi, ma sembra che funzioni e che i bambini siano in grado/capaci di esplorare a modo loro.

Ho notato che loro lasciano ai bambini molta libertà, li lasciano fare [...] noi invece li proteggiamo troppo.

Sembra che i bambini italiani sappiano esattamente ciò di cui hanno bisogno e in quale momento ... senza alcuno sforzo. Mentre io penso molte volte che siano momenti molti impegnativi per le educatrici.

Credo che $\mathrm{i}$ bambini (italiani) sianomolto più autoregolati/autonomi [...] le loroattività non sono poi così diverse dalle nostre.

Alcune scene del filmato americano sollecitano le educatrici italiane a rintracciare ed esplicitare le ragioni del loro stile di intervento prevalentemente indiretto nelle proposte di gioco/attività e si sforzano di motivare le ragioni della loro scelta di non suggerire/dirigere/stimolare troppo, di allestire contesti piuttosto che suggerire condotte. Come afferma un'educatrice vedendo il filmato americano centrato su un'attività con la sabbia:

C'è molto l'intervento dell'educatrice [...]: è lei che decide quali oggetti usare, ed
è molto in mezzo ai bambini, anche durante il gioco. [...] Così il margine di
iniziativa del bambino è molto influenzato. [...] Per noi, invece, sono molto
importanti le scelte che partono dai bambini. Attraverso il confronto interculturale
da un lato si chiariscono le ragioni e si confermano le pedagogie implicite che
orientano l'agire (facciamo cosi perché, ...crediamo sia importante agire cosi per
raggiungere questo obiettivo, ...rivedendomi mi sono confermata... mi sono sentita
parte di un gruppo), dall'altro si amplia la gamma di azioni possibili e degli obiettivi
educativi/curricolari possibili (forse avrei dovuto... forse noi parliamo tropo [...].
[.... Anche i numeri, I'avere tre bambini... probabilmente sono abituati ad avere
un pensiero diverso sull'autonomia.

Il confronto interculturale promuove consapevolezza, attiva processi di pensiero sulle differenze, le somiglianze, la varietà delle attribuzioni di senso e di significato, aiuta a superare interpretazioni stereotipate e onnicomprensive, accogliendo visioni che contengono una pluralità di azioni, ipotesi, finalità.

\section{Parlare altrimenti}

Le parole sono il mezzo con cui i bambini imparano i significati delle cose e con cui le condividono con altri. I bambini e le bambine vivono in contesti di parola e, in quanto partecipanti attivi e attori sociali, contribuiscono attivamente, al pari degli adulti, a definire la qualità e la quantità degli scambi verbali che caratterizzano le loro relazioni 
coni pari e con gli adulti-educatori. Eppure, raramente ci prendiamo una pausa dal parlare quotidiano soffermandoci a pensare a "come parliamo" con i bambini (Bove, 2016). E questo è particolarmente vero nel contesto educativo del Nido dove accadono molte cose contemporaneamente e dove le parole degli adulti e quelle dei bambini ciascuna con i suoi significati - si intrecciano costruendo un ambiente comunicativo specifico di cui tuttavia non sempre siamo consapevoli.

Questo è uno dei temi che è emerso in modo inatteso nei momenti di confronto interculturale che hanno coinvolto le educatrici dei due Nidi contesti della ricerca a cui facciamo riferimento: non era un tema chiave nella ricerca che aveva obiettivi più ampi, nè era stato previsto tra i temi possibili, non era tra i temi di maggior interesse delle educatrici, nè tanto meno rientrava tra le curiosità rispetto all'altro contesto educativo. Eppure, dopo aver visto il filmato delle colleghe americane alle prese con la gestione di un momento di spuntino a metà giornata con i bambini, molte educatrii Italiane si sono soffermate sulle modalità comunicative delle educatrici. Riportiamo una sintesi descrittiva di una clip-video tratta dalle videoregistrazioni della giornata educativa nel nido americano per esemplificare la situazione rappresentata nel filmato e le reazioni delle educatrici.

\begin{abstract}
Clip-15
Siamo nel nido Americano e l'educatrice è seduta al tavolo con un gruppetto di bambini di età compresa tra i12 e i 24 mesi. E' il momento della merenda e, mentre l'educatrice è seduta, distribuisce la merenda: parla rivolgendosi a un bambino/a alla volta, accompagnando le azioni con le parole o frasi esemplificative- quali "ecco il latte, il pane, il pane tostato" - oppure ripetendo le parole dei bambini - oppure ripete le parole dei bambini (quella è la tua sedia? Ti do subito il latte), o usa formule di cortesia (Per favore, aiutami, grazie). Mentre parla, ripete spesso alcune parole soffermandosi sulla pronuncia: per esempio dicendo, in risposta a un bambino che cerca di nominare un particolare tipo di cibo, "Cannella è una parola... difficile". In quasi tutti gli scambi si rivolge a un bambino alla volta.
\end{abstract}

Vedendo questa situazione ripresa nel filmato le educatrici del nido Italiano si sono confrontate sulle modalità con cui la collega americana si rivolge e parla ai bambini, notando in particolare: la dimensione dell'interazione comunicativa per lo più "individualizzata", vale a dire con uno stile comunicativo che vede l'educatrice rivolgersi a un bambino/a alla volta, e l'attenzione al vocabolario e alla precisione nell'uso delle parole. Si tratta di due comportamenti che balzano all'occhio delle educatrici italiane

\footnotetext{
${ }^{5}$ La scena a cui facciamo riferimento è stata discussa anche in un precedente contributo proprio su questo tema a cui rimandiamo per approfondimenti: BOVE C., (2016), "Le parole nella relazione con i bambini: osservare il "linguaggio in azione" al nido", in S. Mantovani, Silva C., Freschi E. (a cura di) Didattica e Nido d'infanzia. Metodi e pratiche di intervento educativo, Junior-Spaggiari, Parma.
} 
che così affermano: "Noi, sempre, ampliamo la discussione al gruppo per tenere tutti i bambini al tavolo in quel momento, per coinvolgerli tutti; per noi lo spuntino è un momento di convivialità" (Educatrice, Italia). E ancora: "[Ho notato] la precisione nell'uso delle parole e la ripetizione della pronuncia" (Educatrice, Italia). Il tema del parlare con i bambini individualmente, favorendo I'interazione comunicativa uno a uno, o del parlare con i bambini come gruppo, costruendo uno scambio verbale multivocale e capace di tenere insieme il gruppo è stato uno dei più discussi nei momenti di confronto interculturale che hanno preso avvio dopo la visione di questo filmato. Allo stesso modo, le educatrici Italiane sono rimaste colpite dall'uso delle parole e dei vocaboli da parte delle colleghe americane, orientato per lo più ad ampliare il vocabolario dei bambini, stimolando sequenze dialogiche basate sulla ripetizione di parole "difficili" (per esempio, dicendo: "Cannella è una parola difficile") e rivolte a sostenere nei bambini l'impegno e lo sforzo della pronuncia corretto in relazione a un particolare tipo di cibo.

Questa breve sequenza video ha dunque funzionato come reattivo e stimolo (TOBIN, MANTOVANI, BOVE, 2010) per far emergere un interessante - e del tutto inatteso - processo di rilettura critica delle proprie abitudini o strategie educative in relazione al linguaggio e all'uso delle parole al nido rendendo visibile quel nesso tra "acquisizione del linguaggio" e "socializzazione linguistica" che è stato messo a fuoco dagli studiosi del linguaggio in contesti culturali diversi (OCHS, 1986, 2006). Se il nesso tra linguaggio e contesto culturale è ben noto in letteratura, si pensi in proposito agli interessanti lavori di ricerca dei teorici dell'etnopragmatica della comunicazione o i teorici del linguaggio in contesti culturali diversi (OCHS, 1986, 2006) da cui si evince il ruolo del linguaggio come strumento o artefatto culturale che media e facilita l'ingresso dei bambini nella comunità culturale, ciò che emerge qui è la necessità di esporre le educatrici che lavorano oggi al Nido alla possibilità di riflettere anche sul proprio ruolo e sulla propria responsabilità educativa rispetto all'acquisizione di un determinato stile comunicativo rispetto ad un altro. Come emerge dalle ricerche etnografiche sull'educazione in comunità culturali diverse (si pensi per esempio alle ricerche di $C$. Snow, 1986, sull'interazione madre-bambino), è il "come" parliamo con i bambini (e non ai bambini) che determina il modo con cui i bambini, a loro volta, useranno e giocheranno con le parole nelle loro interazioni con i pari e con altri adulti. Parlare con un bambino alla volta o con il gruppo in quanto unità, incoraggiare i bambini a pronunciare le parole correttamente, insistere sull'acquisizione di determinati vocaboli, piuttosto che esplicitare i sentimenti e usare le parole come medium per costruire 
relazioni interpersonali empatiche di sintonia, sono tutte strategie educative che emergono in modo evidente in contesti di confronto interculturale - come quelli creati nel corso di questa ricerca - ma che, nella quotidianità, rischiano di rimanere implicite.

Ciò significa, come confermano le ricerche che hanno osservato gli usi linguistici nelle diverse comunità scolastiche in culture diverse (TOBIN, WU, DAVIDSON, 1989; Clancy, 2009), che parlando veicoliamo credenze rispetto alle parole in uso e ai significati che sono propri del contesto in cui agiamo con le parole e che ne determinano la qualità e gli usi. Le norme in uso rispetto a come "parlare" e come "parlarsi" - si pensi per esempio allo stile diretto o indiretto, alla forma individuale o plurale, all'esplicitazione o meno dei sentimenti (per esempio nello stile Giapponese di cui ci parla Clancy nella sua etnografia con madri Giapponesi da cui emerge quanto le madri scoraggino un uso del linguaggio diretto, e incoraggino il silenzi, Clancy (2009, p.263) determinano il nostro modo di usare le parole.

Parole e ambiente sono dunque strettamente interrelati e il vocabolario degli adulti che stanno con i bambini incide profondamente sul vocabolario e gli usi del linguaggio che i bambini imparano. Molte volte questo non sembra un tema prioritario eppure - come è emerso dalla ricerca di cui stiamo parlando - non appena veniamo esposti a modalità diverse di usare le parole, si apre la possibiltà di rivedere criticamente le nostre modalità di usare le parole con i bambini. Ampliare il vocabolario in uso con i bambini, incoraggiarli all'uso di termini precisi, considerare la possibilità di accompagnare i bambini a impadronirsi di abitudini di uso delle parole più preciseordinate, è diventato un tema importante per le educatrici del Nido italiano proprio grazie all'esposizione a un altro-modo di gestire il flusso di parole che caratterizza la quotidianità del Nido.

Parallelamente, anche la visione della sequenza videoregistrata nel Nido italiano ha fatto emergere reazioni interessanti da parte delle educatrici americane che, a loro volta, hanno avuto una funzione di stimolo-indiretto alla riflessione rispetto al valore della dimensione sociale del linguaggio e del tema del gruppo come contesto di socializzazione-linguistica: diversamente dalla scena riportata sopra, nel caso della clipvideo realizzata durante il momento del pasto nel Nido italiano, emerge un modo di parlare più rivolto al gruppo che al singolo bambino o bambina, caratterizzato da espressioni quali: "oggi c'è il riso con i piselli", "A. dice che non gli piace ...e invece M. dice che ...", "Adesso mangiamo, poi ..". Lo stile dialogico sembra essere uno versus molti, vale a dire orientato più al gruppo che al singolo e l'educatrice sembra giocare un ruolo di mediazione e costruzione del gruppo più che di raccolta di parole e richiesta di 
precisazioni. Mentre distribuisce il cibo nei piatti dei bambini, parla con loro usando la forma plurale, rivolgendosi a un bambino ma tenendo sempre la forma espressiva plurale in modo da "costruire un processo di dialogo tra più bambini", allargando il discorso, connettendo quello che dice un bambino a quello che dicono altri.

Il risultato, a detta della stessa educatrice protagonista mentre si rivede, è un traffico di parole fin eccessivo: "quanto parliamo!" osserva l'educarice Italiana dopo aver visto il filmato che la ritrae durante il momento del pasto con i bambini. Emerge il numero di parole e la prevalenza della voce dell'adulto. Sembra cioè che le educatrici si accorgano della quantità e della qualità dell'uso delle parole proprio grazie a questa doppia esposizione culturale: al proprio modo di parlare e di stare con i bambini al Nido attraverso le parole - rappresentata dalla videoregistrazione della propria giornata educativa - e al modo di parlare e di stare con i bambini caratteristico del contesto americano. In qualche caso l'educatrice che si rivede arriva a osservare quanto la propria voce sia eccessiva rispetto a quella dei bambini e delle bambine: una voce che occupa un tempo esteso, prolungato, che mentre accompagna la costruzione del gruppo sembra togliere spazio al tempo verbale dei bambini.

Pensando alla dimensione multilinguistica dei contesti educativi contemporanei, predisporre dispositivi per osservare-osservarsi anche a partire dall'uso delle parole o dalle parole in uso che veicoliamo nei contesti per l'infanzia è un tema importante in un momento in cui gli educatori e le educatrici sono chiamati a sviluppare strategie e acquisire competenze per il dialogo multivocale e interculturale. A prescindere dalle lingue, anche le simbologie e i concetti veicolati dalle parole e dal come queste sono usate sono oggi oggetti educativi importanti che devono rientrare nella progettazione dei luoghi di vita per i bambini e le bambine fin dalla prima infanzia.

L'aver messo la parola in uso al centro della riflessione interculturale mediata dalle videoregistrazioni, illustrata dall'esempio di ricerca oggetto di questo articolo, è stato un modo per ampliare la prospettiva delle educatrici e le relative strategie verbali. Un esercizio di antropologia dialogica e di dialogo interculturale rispetto a un tema - le parole - spesso considerato talmente parte della vita quotidiana nei contesti educativi da passare inosservato. Oggi la formazione linguistica avviene in contesti multilinguistici: il che, a maggior ragione, richiama alla responsabilità degli educatori rispetto alla propria formazione e alla maturazione di consapevolezze rispetto alle parole veicolate nei contesti di vita quotidiani.

\section{Alcuni risultati e prospettive}


La ricerca a cui abbiamo fatto riferimento fa emergere due aspetti su cui vorremmo richiamare I'attenzione in quanto dispositivi metodologici che accelerano il confronto interculturale: l'importanza di combinare la dimensione individuale e di gruppo della riflessione sulle pratiche educative intrecciando descrizione e confronto sui significati e la scrittura come pratica riflessiva e premessa al confronto dialogico, allo scambio e al confronto con gli altri. Come il dialogo interculturale sembra ampliarsi quando si alternano momenti di riflessione individuale e di gruppo, così il confronto è tanto più generativo (di prospettive, idee e strategie di intervento) quanto più è preparato da un dialogo "autentico" innanzitutto con se stessi/con le premesse che orientano il pensare e l'agire educativo di ciascuno. Il dialogo con se stessi funge da organizzatore delle intenzioni o credenze che orientano l'azione e prepara in tal senso alla condivisione. Così concepito, il lavoro sull'osservazione intrecciato a quello sui significati traduce molto concretamente l'immagine dell' "insegnante-investigatore" (DEWEY, 1910, 1933) che rileva, progetta, verifica e che non può essere un mero esecutore di un programma.

Anche le acquisizioni scaturite dalle difficoltà che abbiamo incontrato nel percorso di ricerca si sono rivelate delle risorse. L'uso delle tecnologie multimediali, che da un lato ci ha consentito di affrontare i problemi dell'archiviazione dei dati video, del dialogo a distanza e del confronto interculturale, dall'altro ha reso evidente la necessità di sviluppare nuove competenze connesse alla produzione, al montaggio e allo scambio e analisi dei materiali video (dai problemi etici connessi alla tutela della privacy che il video amplifica, alla difficoltà di lavorare su materiale video in streaming, alla gestione del tempo nei collegamenti via skype e videoconferenze, all'uso di più telecamere, microfoni per la registrazione dei dialoghi, eccetera). Anche la differenza linguistica funge da ostacolo, ma nel contempo da "rallentatore utile" (BRAGA, 2009) che in parte struttura il pensiero e spinge al nucleo essenziale dello scambio: il filtro della traduzione, talvolta anche i fraintendimenti linguistici, obbligano ad esplicitare, chiarire e chiarirsi presupposti, impliciti, cornici. Utili esercizi di esplicitazione (di presupposti, cornici, riferimenti teorici, accezioni, interpretazioni, orizzonti di significato) che periodicamente potrebbero avvenire anche tra gruppi di lavoro di uno stesso contesto, tra operatori di servizi diversi: siamo così sicuri di intendere tutti la stessa cosa quando parliamo di «dare regole a un bambino», «sostenere I'acquisizione di autonomia», «accogliere un genitore»? 
Questa ricerca, ma anche le sempre più diffuse esperienze di "gemellaggi" consolidate anche in Italia e in crescita negli ultimi anni, ha confermato la fertilità delle esperienze di scambi pedagogici tra realtà e paesi diversi, pratiche di allenamento al decentramento inteso come competenza trasversale cruciale/necessaria al lavoro con i bambini e le famiglie. Oggi l'uso di video digitali e di tecnologie per la comunicazione a distanza rende queste esperienze di scambio e dialogo inter e cross culturale più agili, flessibili e sostenibili, ma richiede la messa a punto di dispositivi e metodi pensati, sistematici e rigorosi per non disperdere questa potenzialità.

Interculturale è anche la dimensione del confronto tra due servizi della stessa città o di città diverse, o tra due sezioni dello stesso nido, o tra educatrici e studenti/tirocinanti. Non si deve necessariamente andare in contesti lontani, come bene evidenzia la ricerca antropologica, per esercitarsi al decentramento, vedere i fenomeni da altri punti di vista: "...Noi diamo per scontate attività e definizioni, a meno che non abbiamo l'opportunità di osservarle da una posizione strategica al di fuori della nostra società" (ROGOFF 1990, p.225).

Non è necessario filmare una giornata intera e avviare impianti e percorsi complessi come quello che abbiamo descritto per promuovere esperienze di indagine, rilevazione, osservazione sistematica e riflessione sui significati: si possono utilizzare sequenze più brevi, videoregistrazioni di episodi circoscritti, esplicitando le ragioni della scelta di una particolare situazione e di un particolare contenuto/tema educativo, annotando le ragioni per cui si è interessati a discuterlo e documentando il percorso di analisi e discussione del materiale osservativo che si è scelto di condividere. Oppure si possono attivare scambi di breve sequenze filmate tra due educatrici della stessa sezione, di sezioni diverse, di nidi diversi della stessa città ma anche di città diversetra servizi diversi (per esempio tra nidi e scuole dell'infanzia): anche i "gemellaggi" tra servizi della stessa città attivano processi "interculturali" e possono essere preziose occasioni di confronto allargato che fungono da "terzo occhio" per rileggere le proprie pratiche ed "esplicitare quelle ragioni del proprio agire educativo che tanto spesso rimangono sottintese nello scambio professionale tra insegnanti durante il lavoro quotidiano" (GIOVANNINI et al., 2018, p.62).

Stiamo monitorando anche alcune ricadute interessanti che questo percorso ha avuto sulle pratiche di tutoraggio delle educatrici nuove e delle tirocinanti (coinvolte in entrambi i contesti in quanto nidi universitari e dunque laboratori di formazione) e sulle possibili trasposizioni di alcuni strumenti e suggestioni metodologiche nella formazione in servizio delle educatrici più giovani. Abbiamo avviato processi di osservazione e 
video-feedback in coppia mediati dal ricercatore o dal coordinatore, sia nei servizi educativi, sia nella didattica universitaria. Si tratta di un lavoro di confronto a coppie tra educatrici nel contesto del Nido (tra educatrice esperta e più giovane o tra educatrici giovani, o educatrice tutor e tirocinante, ecc.) centrato sulla revisione dapprima individuale e poi reciproca di brevi filmati (o di osservazioni carta e matita) dove il confronto è mediato da alcune domande stimolo iniziali e in itinere (MORAN, 2009). Anche in questo caso è sufficiente scegliere una breve sequenza su una situazione o momento "caldo" che le educatrici coinvolte ritengono significativo.

Sempre in collaborazione con i colleghi delle due Università, abbiamo sperimentato, anche se ancora in fase pilota, alcune esperienze di scambio crossculturale che hanno coinvolto alcuni studenti dei nostri Corsi di Laurea mediate dalle tecnologie ${ }^{6}$. Si tratta di un percorso basato sul confronto tra coppie di studenti e dottorandi di paesi diversi che, a distanza, collaborano nella messa a punto di un ipotetico progetto di ricerca (sulla scia delle esperienze di critical friends, BASKERVILLE, GOLDBLATT (2009)). La valenza formativa e la generatività di questi percorsi si evince dalle considerazioni dei partecipanti che, in sede di verifica, hanno sottolineato le loro acquisizioni e nuove consapevolezze:

Collaborare, attraversando differenti registri linguistici e culturali, penso abbia rivelato alcune mie modalità nello stare concretamente in un gruppo, mi sono potuta domandare in che maniera mi sono adattata al gruppo e quali strategie ho messo in atto vivendo le conversazioni on-line con le mie compagne di ricerca. Questa esperienza mi ha permesso di riflettere sulle mie particolari modalità di integrazione all' interno del gruppo, sulle mie possibili resistenze e sulle aperture che mi caratterizzano. Confrontarsi con I'altro implica inevitabilmente un lavoro su se stessi: questo accade in maggior misura quando si entra in relazione con persone straniere che possiedono diversi modelli culturali e parlano una lingua differente" (Studentessa, Italia).

My take- away (so far): cross cultural research adds so many complexities that I really didn't understand until I had to deal with them; (...) Working with someone who has differences has brought a new perspective and inspired new ideas. (Studentessa, Tennessee).

Credo che questo percorso abbia inciso decisivamente sulle mie risorse personali, riuscendo a stimolarmi da diversi punti di vista. Ho riflettuto sul "sistema" del quale faccio parte, cercando anche di estraniarmi riuscendo così a vederlo con altri occhi. Non avrei mai pensato, arrivata a questo punto di aver arricchito così tanto il mio patrimonio grazie questa esperienza. Mi è servito per credere in me e tentare riuscendo (valutazione personale rispetto le mie iniziali aspettative) a superare difficoltà che pensavo sarebbero state molto più difficili da superare. (Studentessa, Italia).

What I learned: Italy is 6 hours ahead of us now, not 7 . For 3 meetings, I thought that Alessandra was late to the Zooms because she was on "Italian time". OOPS! If you're not an expert, make yourself one. There is no way to "plan" a relationship

6Progetto: «Cross-Cultural Perspectives in Early Care and Education» (Scientific Supervisor: Mary Jane Moran, in collaboration with Chiara Bove, Piera Braga, 2015) 
in cross-cultural research. You don't have to force a relationship with your partner...the relationship kind of just happens" (Studentessa Tennessee).

L'intercultura è un amplificatore degli aspetticomunicativi, delle loro caratteristiche, ma anche delle cautele, degli accorgimentiche ci possono aiutare a raggiungere una comunicazione piùfluida ed efficace e ad aprire prospettive nuove (... "to consistently reflect upon ethnocentrism" per usare le parole di una studnetessa americanacoinvolta nelpercorso). In alcuni casi questipercorsi di scambio a distanzacentratisulla co-costruzione di un ipoteticoprogetto di ricercahannoattivatoprocessi di negoziazione, in altricasiqualcosa di più simile a un compromesso. Il dover «scendere a patti», trovare una «strada di mezzo, cheunisca me e l'altro» puòessere visto come un'acquisizione o una debolezza: se lo vediamo come una debolezza, saremoportati a sentire di «aver dovutocedere» rispetto allenostreintenzioni; se invece lo vediamo come un'acquisizione, «indietreggiare rispetto alla propria posizione» è visto come «fare un passoavanti per la costruzione di qualcosa di condiviso».

Alla luce delleacquisizioni maturate nelcorsodellaricerca e deisuoisviluppianche in altriprocessi di formazione, come abbiamoaccenato fin qui, stiamosperimentando la trasposizione di alcunistrumenti in pratiche formative sostenibili in presenza ma anche a distanza, combinandometodivisuali, testuali, narrativi.

\section{PER CONCLUDERE}

I video, abbiamo visto fin qui, sono strumenti potenzialmente molto interessanti per la formazione degli educatori e degli insegnanti, come ampiamente discusso in letteratura. L'esperienza di ricerca a cui abbiamo fatto riferimento in questo articolo, conferma quanto questo potenziale cresca quando le immagini video sono utilizzate in contesti di formazione inter e cross-culturale. In questo caso, infatti, le immagini diventano lo stimolo che apre possibilità di sviluppo di competenze interculturali oggi sempre più necessarie per lavorare in contesti educativi di qualità.

Lo scambio mediato dalle immagini, costruite ad hoc per tali fini, può essere organizzato in molti modi e contesti: può avvenire in modalità sincrona, asincrona, a distanza di tempo, a più riprese, coinvolgendo uno, due o più interlocutori. Può fungere da stimolo per aprire il dialogo in gruppi omogenei, ma anche eterogenei. Ciò che aiuta è l'immagine che funge da ancoraggio a un tema: rispetto al solo scambio verbale, dove a volte è difficile per il gruppo "stare sul tema", metterlo a fuoco e circoscriverlo, 
l'immagine cattura lo sguardo e aiuta a focalizzare l'attenzione sulle questioni più rilevanti. Se poi l'immagine è anche "tecnicamente" bella, piacevole, divertente, il confronto è ancora più efficace, come abbiamo avuto modo di imparare anche in ricerche precedenti $\mathrm{e}$ in particolare in percorsi di video-etnografia (TOBIN, HSUEH, 2009).

Un approccio e uno strumento, dunque, interessante per la formazione di una postura interculturale fin dall'infanzia su cui vale la pena continuare a investire in termini di studio e di ricerca.

Ci piace chiudere questa riflessione con le parole di Barbara Rogoff che ben sintetizzano la valenza formativa dello spiazzamento culturale discussa fin qui:

\begin{abstract}
Non esiste 'un modo migliore' di fare le cose. Studiare le diverse pratiche culturali non significa determinare quale sia 'giusta' (e con questo non intendo dire che tutte le pratiche siano giuste e positive). $\mathrm{E}^{\prime}$ richiesto un atteggiamento di apertura a diverse possibilità, che non si escludono necessariamente a vicenda. Apprendere da altre culture non significa rinunciare alle proprie tradizioni e pratiche, bensì sospendere momentaneamente le proprie certezze e separare con cura lo studio delle pratiche culturali da giudizi di valore. $E^{\prime}$ fondamentale elaborare una serie di ipotesi sui modelli osservati, e nel contempo verificarle e rivederle continuamente a mente aperta. C'è sempre qualcosa da imparare (ROGOFF 2003, p.378).
\end{abstract}

\title{
REFERÊNCIAS
}

ALTET, Marguerite;CHARLIER,Évelyne; PAQUAY,Léopold; PERRENOUD, Philippe. (orgs.) Formare gli insegnanti professionisti. Quali strategie? Quali competenze? Roma: Armando, 1996.

ANOLLI, Luigi. La sfida della mente multiculturale. Nuove forme di convivenza. Milano: Raffaello Cortina, 2011.

BASKERVILLE, Delia; GOLDBLATT, Helen. Learning to be a critical friend: from professional indifference through challenge to unguarded conversations. :.

Cambridge Journal Of Education, [s.l.] , v. 39, n. 2, p. 205-221, 27 maio 2009. Informa UK Limited. http://dx.doi.org/10.1080/03057640902902260.

BRUNER, Jerome S. La cultura dell'educazione. Nuoviorizzonti per la scuola, Milano: Feltrineli, 1997[1996].

BOVE, Chiara; SHARMAHD, Nima. Beyond invisibility. Welcoming children and families with migrant and refugee background in ECEC settings. European Early Childhood Education Research Journal, [s.I.], v. 28, n. 1, p. 1-9, 2 jan. 2020. Informa UK Limited. http://dx.doi.org/10.1080/1350293x.2020.1707940.

BOVE, Chiara; JENSEN, Bente; WYSłOWSKA, Olga; IANNONE, Rosa Lisa; MANTOVANI, Susanna; KARWOWSKA-STRUCZYK, Małgorzata. How does innovative continuous professional development (CPD) operate in the ECEC sector? Insights from a crossanalysis of cases in Denmark, Italy and Poland. European Journal Of Education, 
[s.I.], v. 53, n. 1, p. 34-45, 22 jan. Wiley. 2018.

http://dx.doi.org/10.1111/ejed.12262.

BOVE Chiara. Ricerca educativa e formazione. Contaminazioni metodologiche. Milano: Franco Angeli, 2009.

BOVE, Chiara. Prospettive di pedagogia culturale nei servizi per l'infanzia. Studium Educationis, Padova, v. 3, n. , p. 91-104, out. 2012. Disponível em:

https://ojs.pensamultimedia.it/index.php/studium/article/view/552/534 Acesso em: 24 abr. 2020.

BOVE, Chiara; BRAGA, Piera. Il Nido come laboratorio di ricerca: osservare e osservar(si) nei contesti educativi per l'infanzia. In: ZANINELLI, Francesca L. (a cura di).Contesti e temi educativi zero-tre anni. Parma : Junior- Spaggiari edizioni srl, p.273-295. 2014.

BOVE, Chiara; BRAGA, Piera; CESCATO, Silvia. Semantiche personali e processi riflessivi. Analisi di una storia di formazione. Studium Educationis, Padova, anno XV, n. 1, p. 75-84, fev. 2014.

BOVE, Chiara., BRAGA, Piera. MANTOVANI, Susanna. "Videoricerca e formazione. Un approccio interculturale". In:BOBBIO, Andrea., TRAVERSO, Aandrea. (a cura di)

Contributi per una pedagogia dell'infanzia Teorie, modelli, ricerche. Pisa: ETS edizioni, P.147-173. 2016.

BOVE, Chiara.Le parole nella relazione con i bambini: osservare il "linguaggio in azione" al nido. In: MANTOVANI, Susanna;SILVA, Clara;FRESCHI, Enrica (a cura di).

Didattica e Nido d'infanzia. Metodi e pratiche di intervento educativo. Parma: Junior-Spaggiari, p. 25-58. 2016.

BRAGA, Piera. (cura di), Promuovere consapevolezza. Esperienze di formazione tra ricerca e pratica educativa. Bergamo: Junior, Azzano San Paolo. 2009.

BRAGA, Piera; MANTOVANI, Susanna. "L'asilo nido come 'laboratorio di formazione'. Ricerca e sperimentazione formativa nel nido Bambini Bicocca". In: ONGARI, Barbara; TOMASI, Francesca(a cura di), Nido d'infanzia 5. Prospettive di ricerca e spunti per la formazione. Trento:Erickson, p.93-112. 2012.

BRAGA, Piera; MANTOVANI, Susanna. Il Nido Bambini Bicocca. Nidi D'ínfanzia, n. 3, p. 32-35, Jan./ Fev. 2017.

BRAGA, Piera; BOVE Chiara. "Osservare e osservarsi al nido". Bambini, n.8, p.20-23, Ottobre, 2014.

CESCATO, Silvia; BOVE, Chiara; BRAGA, Piera. Video, formazione e consapevolezza. Intrecci metodologici. Form@re: Open Journal per la formazione in rete, Firenze, v. 15, n. 2, p. 61-74, 2015.

CLANCY, Patrica M. "L'acquisizione dello stile comunicativo nei giapponesi". In:

LEVINE, Robert A.; NEW, Rebecca s. (Eds.), Antropologia e infanzia. Sviluppo, cultura, educazione. Milano: R. Cortina, p. 263-291. 2009 [2008]. 
COMMISSIONE EUROPEA. Educazione e cura della prima infanzia: consentire a tutti i bambini di affacciarsi al mondo di domani nelle condizioni migliori. Bruxelles, 2011, http://eurex.europa.eu/legalcontent/IT/TXT/PDF/?uri=CELEX:52011XG0615(04)\&from=IT.

EUROPEAN COMMISSION. "Communication 'Investing in Children: Breaking the Cycle of Disadvantage'." Official Journal European Commission 59: 5-16, 2013. http://data.europa.eu/eli/reco/2013/112/oj. [Google Scholar]

COMMISSIONE EUROPEA. Proposal for key principles of a Quality Framework for Early Childhood Education and Care, OECD Publishing, 2014.

DEL BOCA, Daniela.Child Poverty and Child Well-Being in the European Union: policy overview and policy impact analysis. A case study: Italy. Budapest \& Brussel: TARKI- Applica, 2010.

DEWEY, John. Come pensiamo, trad. it. a cura di ChiaraBove. Milano:Raffaello Cortina Editore, 2019[1933].

GIOVANNINI, Donatella; MUSATTI,Tullia., PICCHIO, Mariacristina.

Incrociareglisguardisull'espereinzadei bambini: documentare, riflettere, narrare". In:MUSATTI, Tulliaet.al. Stare insieme. Conoscere insieme. Bambini e adulti nei servizi educativi per l'infanzia. Parma: Junior, 2018.

GOLDMAN,Ricki;PEA,Roy;BARRON,Brigid;DERRY,ShoronJ. (a cura di), Videoricercaneicontesti di apprendimento.Teorie e metodi, trad.it. Milano: Raffaello Cortina, Milano, 2009 [2007].

LEVINE, Robert A.; NEW, Rebecca s. (Eds.), Antropologia e infanzia. Sviluppo,cultura, educazione. Milano: R. Cortina,2009 (2008).

MANTOVANI, Susanna; BRAGA,Piera; GAMBINI, Annastella. "Incontrare i viventi. Esperienze scientifiche al Nido" In: MANTOVANI, Susanna; SILVA, Clara; FRESCHI, Enrica (a cura di). Didattica e Nido d'infanzia. Metodi e pratiche di intervento educativo. Parma: Junior-Spaggiari, p.59-84. 2016.

MANTOVANI, Susanna; SILVA, Clara; FRESCHI, Enrica (a cura di). Didattica e Nido d’infanzia. Metodi e pratiche di intervento educativo. Parma: Junior-Spaggiari, 2016.

MANTOVANI, Susanna.Italy: Pedagogy. In: NEW, Rebecca Staples;COCHRAN, Moncrieff; (eds.), Early childhood education: an international encyclopedia, vol. 4,Westport:Praeger, p. 1115-1118. 2007.

MORAN, Mary. J.Apprendimento collaborativo e uso della fotografia come metodo visuale: un esempio americano". In: BOVE,Chiara.Ricerca educativa e formazione. Contaminazionimetodologiche. Franco Angeli, Milano, p. 247-270. 2009.

MORAN, Mary Jane; BOVE, Chiara; BROOKSHIRE, Robyn; BRAGA, Piera; MANTOVANI, Susanna. Learning from each other: the design and implementation of a cross-cultural research and professional development model in italian and u.s. toddler classrooms: The design and implementation of a cross-cultural research and professional development model in Italian and U.S. toddler classrooms. Teaching And Teacher 
Education, [s.I.], v. 63, p. 1-11, abr. 2017. Elsevier BV. http://dx.doi.org/10.1016/j.tate.2016.10.018.

MORAN, Mary J.; BROOKSHIRE, Robyn; BOVE, Chiara; BRAGA Piera; MANTOVANI, Susanna. "Co-Constructed Research Design: Lessons on Equivalency and Teacher Participation in a US-Italian Professional Development Study". In: APKOVO, Samara M.;MORAN, Mary J.;BROOKSHIRE, Robyn. (eds). Collaborative Cross-Cultural Research Methodologies in Early Care and Education Contexts, Devon: Taylor\& Francis, 133-149, New York. 2018.

OCHS, Elionor. From feeling to grammar: a Samoan case study. In: SCHIEFFELIN, Bambi B.; OCHS, Elionor. (eds.), Language socialization across cultures. Cambridge: Cambridge University Press, p. 251-272. 1986.

OCHS, Elionor. Linguaggio e cultura. Roma: Carocci, 2006.

OECD The resilience of students with an immigrant background: Factors that shape well-being. Paris: OECD Publishing, 2018.

ROGOFF, Barbara. Imparando a pensare. L'apprendimento guidato nei contesti culturali.Milano: Raffaello Cortina, 2006 [1990].

ROGOFF, Barbara. La naturaculturaledellosviluppo. Milano: Raffaello Cortina, 2004 [2003].

SNOW C.E. Parlare con i bambini. In: FLETCHER, P.; GARMAN, G. L'acquisizione del linguaggio. Milano: Raffaelo Cortina, Milano, p. 105-132. 1991 [1986].

TOCHON F.V. Dai video-casi alla video-pedagogia. Una cornice teorica per il videofeedback e la riflessione con i video nella pratica della ricerca pedagogica. trad. it. In: GOLDMAN, Ricki; PEA, Roy; BARRON,Brigid; DERRY, Sharon J.Videoricerca nei contesti di apprendimento. Teorie e metodi. Milano: Raffaello Cortina Editore, 2009[2007].

TOBIN, Joseph J.; WU, David Y. H.; DAVIDSON, Dana H. Infanzia in tre culture. Giappone, Cina e Stati Uniti. Milano: Raffaello Cortina, 2000 [1989].

TOBIN, Joseph J., HSUEH, Yeb; KARASAWA, MAYUMI. Infanzia in tre culture vent'anni dopo, Milano: Raffaello Cortina, 2011 [2009].

TOBIN, Joseph J.; MANTOVANI, Susanna; BOVE, chiara. Methodological Issues in Video Based Research on Immigrant Children and Parents in Early Childhood Settings. In:TAROZZI, Massimliano; Mortari, Luigina (ed. by), Phenomenology and Human Research Today. Bucarest: Zeta Books, Bucarest, p. 204-225. 2010. 


\section{NOTAS}

RECIPROCAL LEARNING: IL CONFRONTO INTERCULTURALE COME DISPOSITIVO PER LA FORMAZIONE DEGLI EDUCATORI. INSIGHTS DA UNA RICERCA TRA ITALIA E STATI UNITI. Reciprocal learning: intercultural exchange as a tool for continuing professional development of ECEC's educators - Insights from a research in Italy and in the USA Reciprocal learning: o confronto intercultural como dispositivo para a formação de educadores Insights de uma pesquisa entre Itália e Estados Unidos

Piera Maria Braga Dipartimento di Scienze Umane per la Formazione "RICCARDO MASSA" Università degli Studi di Milano-Bicocca Milano, Italia

piera.braga@unimib.it

(1) $\underline{\text { https://orcid.org/0000-0002-9295-6362 }}$

Chiara Maria Bove Dipartimento di Scienze Umane per la Formazione "RICCARDO MASSA" Università degli Studi di Milano-Bicocca Milano, Italia

chiara.bove@unimib.it (1) https://orcid.org/0000-0003-4818-3777

Mary Jane Moran Department Child and Families Studies University of Knoxville

Tennessee, USA mmoran2@utk.edu

(1) https://orcid.org/0000-0003-1882-4326

Robyn Brookshire

Direttore dell'Early Learning Center for Research and Practice University of Knoxville Tennesee, USA rbrooks8@utk.edu

Ohttps://orcid.org/0000-0001-9525-2865

Endereço de correspondência do principal autor

U16, Piano: IV, Stanza: 7B. Milão, Italia

\section{AGRADECIMENTOS}

Não se aplica.

\section{CONTRIBUIÇÃO DE AUTORIA}

Concepção e elaboração do manuscrito: P. Braga; C., Bove

Coleta de dados: P. Braga, C. Bove (Italy), M.J. Moran, R. Brookshire (USA)

Análise de dados: L P. Braga, C. Bove, M.J. Moran, R. Brookshire

Discussão dos resultados: P. Braga, C. Bove, M.J. Moran, R. Brookshire

Revisão e aprovação: P. Braga; C., Bove

\section{CONJUNTO DE DADOS DE PESQUISA}

O conjunto de dados que dá suporte aos resultados deste estudo não está disponível publicamente.

\section{FINANCIAMENTO}

Não se aplica.

\section{CONSENTIMENTO DE USO DE IMAGEM}

Não se aplica.

APROVAÇÃO DE COMITÊ DE ÉTICA EM PESQUISA 
Não se aplica.

\section{CONFLITO DE INTERESSES}

Não se aplica.

LICENÇA DE USO - uso exclusivo da revista

Os autores cedem à Zero-a-Seis os direitos exclusivos de primeira publicação, com o trabalho simultaneamente licenciado sob a Licença Creative Commons Attribution (CC BY) 4.0 International. Esta licença permite que terceiros remixem, adaptem e criem a partir do trabalho publicado, atribuindo o devido crédito de autoria e publicação inicial neste periódico. Os autores têm autorização para assumir contratos adicionais separadamente, para distribuição não exclusiva da versão do trabalho publicada neste periódico (ex.: publicar em repositório institucional, em site pessoal, publicar uma tradução, ou como capítulo de livro), com reconhecimento de autoria e publicação inicial neste periódico.

PUBLISHER - uso exclusivo da revista

Universidade Federal de Santa Catarina. Núcleo de Estudos e Pesquisas da Educação na Pequena Infância - NUPEIN/CED/UFSC. Publicação no Portal de Periódicos UFSC. As ideias expressadas neste artigo são de responsabilidade de seus autores, não representando, necessariamente, a opinião dos editores ou da universidade.

EDITORES - uso exclusivo da revista

Márcia Buss-Simão e Kátia Agostinho.

HISTÓRICO - uso exclusivo da revista

Recebido em: 01-05-2020 - Aprovado em: 01-07-2020 